\title{
Harmful Effect of Commonly Used Electronic Devices on Adolescence and its Safeguard at Shebin El-Kom
}

\author{
Dr. Nagwa A. Zein El Dein \\ Ass. Prof. Pediatric nursing Menoufia University
}

\begin{abstract}
:
Purposes of this study were to assess the effects of electronic devices on the health of adolescence and to provide guideline to safeguard them from harmful effect on their health for parents and practitioners Design. Across section study was utilized to collect data from a sample of 59 boys and 67 girls of adolescence students. Age between 16-18 yrs. through Two Tools. the $1^{\text {st }}$ is -adapted Play and Technology Questionnaire for alder children, the $2^{\text {nd }}$ is an interview questionnaire related to harmful effect of commonly used electronic devices on their health, it classified into mild, moderate or severe effects. Results presented an adolescence were expose to moderate to severe hazards as backache, carpal tunnel syndrome, itchy eyes, and sleeping problems which lead to lack of concentration, which effect on their school performance. So, nurses should write guided instruction for parent and teachers to be conducted with adolescence and followed by practitioners, with continuing medical and nursing educational programs to be planned for safeguard their health .
\end{abstract}

Key Wards: Adolescence. Electronic devices, Harmful effect, Safeguard, Nurses, Health practitioners

\section{Introduction}

Electronic devices is an integral part of adolescence's lives in the twenty-first century. The world of electronic devices, however, is changing dramatically. Television, which dominated the media world through the mid-1990s, now competes in an arena crowded with cell phones, computers, iPods, video games, instant messaging, interactive multiplayer video games, virtual reality sites, Web social networks, and e-mail. Gentile, et,al. (2004)

Debates on the effects of new technology have recurred especially with regard to the effect on young people. Each new devices technology brought with it great promise for social and educational benefits, and great concern for adolescence's exposure to inappropriate and harmful content or health hazards. Ellen, (2000)

Adolescents, in particular, spend a significant amount of time viewing and interacting with electronic devices in the form of TV, video games, music, and the Internet. Considering all of these sources together, adolescence spend more than 6 hours per day using media. Nearly half of that time is spent watching TV, playing, or studying with computer.. The remainder of the time is spent using other electronic media alone or in combination with TV (Gentile and Walsh 2002;)

Youth are creative in their uses of new technologies, and this creativity can lead to angst for parents, teachers and health care providers. Teens can download violent videos, send sexual text messages or explicit self-photographs to their friends, but it is unclear whether or how electronic effects or how cognitive processing may be affected. Some neuroscientists worry about the impact of all of this new technology on the developing adolescent brain. The future of children (2000)

In Egypt, there are 83,4 million use Mobile phone, 29,00211 million used the internet access, 1,8 million from them used the ADSL internet, there are 10000 used the internet from the mobile as well as there are 10,7 million used USB Modems. On the other hand, there are 8,7 million used the face book. So Egypt rated as the first class in using this website ( Information Center, El Ahram Journal,2012)

The numbers of adolescence joining social networking websites like Face book and You tub grow daily. Technological convergence, a hallmark of electronic use today, enables youth to access the same source from different devices, often portable, media platforms Jeanne, and Elisabeth,(2008).

Over the long term, computer can cause physical damage. Using a mouse and keyboard for many hours every day can lead to repetitive stress injuries. Back problems are common among people who spent a lot of time sitting at computer desks. Late-night computer sessions cut into much-needed sleep time. Long-term sleep deprivation causes drowsiness, difficulty concentrating, and depression of the immune system. In addition to, when someone spends hours at a computer is obviously not getting any meaningful exercise, So, computer can indirectly lead to poor overall physical condition and even obesity.Playing for long periods of time on the computer can strain adolescence eyes or can worsen existing eye conditions. Also, symptoms include eye discomfort, headaches, itchy eyes and difficulty in focusing. So, It is important to rest the eyes while working on the computer. Unfortunately, it also more prone to postural, muscular and skeletal disorders like tendonitis, 
nerve compression and carpal tunnel syndrome. Plus, some of the special effects of video games may induce seizures. Grabianowski, (2012)

Research has documented negative effects of video games on adolescence's physical health, including obesity, video-induced seizures and postural, muscular and skeletal disorders, such as tendonitis, nerve compression, and carpal tunnel syndrome as well as delayed school achievement. However, these effects are not likely to occur for most adolescence. The research to date suggests that parents should be most concerned about two things: the amount of time that adolescence play, and the content of the what to be play or watching. Brad and Rowell, (2001)

In addition, symptoms associated with using mobile phones most commonly include headaches, earache, warmth sensations and sometimes also perceived concentration difficulties as well as fatigue However, over exposure to mobile phone use is not currently known to have major health effects. Another aspect of exposure is ergonomics. Musculoskeletal symptoms due to intensive texting on a mobile phone have been reported and techniques used for text entering have been studied in connection with developing musculoskeletal symptoms. The central factors appearing to explain high quantitative use were personal dependency, and demands for achievement and availability originating from domains of work, (Sara and Mates, 2011)

To protect adolescence from harm, all health staff must have the competences to recognize adolescence maltreatment and to take effective action as appropriate to their role. They must also clearly understand their responsibilities, and should be supported by their employing organization. In addition, Parents have no idea about electronic devices effects on adolescence. So, parents need to understand that electronic devices can have an impact on everything they're concerned about with their adolescence's health and development, school performance, learning disabilities, sex, drugs, and aggressive behavior. . Jean and Elishabeth,2008)

Professionals are in an excellent position to advocate on behalf of adolescence and their careers to encourage them to take into consideration the rate of change of these technologies. The core task of this statement is to highlight the underlying issues of how both current and future technologies, and the content they deliver, impact on their health and behaviors.

So, nurses should be capable to fulfill their duties and examine links between use of electronic and its harmful effect. Woodard and Gridina,.(2000) .

\subsection{The Framework of safeguard identifies six levels of competence, are as follows:}

- Level 1: Non-clinical staff not working in health care settings as social worker, teachers, school directors

- Level 2: Minimum level required for clinical staff who have some degree of contact with adolescence as radiologist, pharmacists, opticians, audiologist which discover any signs of abused and recognize the impact of electronic in different body system

- Level 3: Clinical staff working with adolescence, and/or their parents/cares and who could potentially contribute to assessing, planning, intervening and evaluating the needs and advising adolescence

and parenting this including general practitioner, school nurse, and health visitors,

- Level 4: Named professionals which in charge for responsibilities during any complain

- Level 5: Designated professionals who demonstrate advanced knowledge on the national safeguard practices and strategies across the health of the community as well as they should aware of latest research evidence and the implications for practice

- Level 6: Experts who gives clinical and health services policy advice in their specialty area to government or other national bodies So, To protect adolescence from harmful effect of electronics all health staff must have the competences to

1-Recognize adolescence at risk and to take effective action as appropriate to their role.

2-Clearly understand their responsibilities, and should be supported by parents to fulfill their duties. Royal

\section{College of Pediatrics and Child Health (2010), \\ 1.2.Theoretical framework}

Electronic devices affect adolescence not only by displacing time they spend doing homework or sleeping but also by influencing beliefs and behaviors and physical health. According to social learning theory, adolescents learn by observing and imitating what they see on the screen, particularly when these behaviors seem realistic or are rewarded. by their peers Bandura (1994) In addition, media present youth with common "scripts" for how to behave in unfamiliar situations such as romantic relationships specially for those age group Tolman et ,al, (2007) added that super peer theory states that the electronic devices are like powerful best friends it sometimes making risky behaviors seem like normative behavior. As well, Social Learning Theory predicts that adolescence can learn new behaviors from television and other audiovisual devices by imitation. Strasburger et, a l (2009)' With the variety of theories suggesting a potentially powerful effect of the electronic 
devices and the growing empirical evidence for negative impact, one might hypothesize that parents would take care to limit exposure to detrimental electronic devices content and eliminate health hazards

\subsection{Operational definitions}

1-Adolescence

As all those who have not yet reached their $19^{\text {th }}$ birthday.

\section{2-Electronic devices}

Defined as any object or process of human origin that can be used to convey media as books, films, mobiles, television, and the Internet. With respect to education ,communication or play. (Tomas, 1998)

3-Safeguarding (adolescence protection)

The term safeguarding is defined as protecting adolescence from maltreatment; preventing impairment of adolescence health or development; ensuring that adolescence are growing up in circumstances consistent with the provision of safe and effective care; Also safe guards are a combination of skills, knowledge, attitudes and values that are required for safe and effective practice Sara and Christina (2008).

\section{Aims of the study}

1-Assess types of electronic devices used by adolescence in Shebin El Kom

2- Assess the health harmful effect of electronic devices produced on adolescence

3-Identify safeguards used by parents and provide suggested guides to safeguard adolescence

\section{Research question}

1- Is there a harmful effect on adolescence from their use of electronic devices

2-Is there a relationship between type and time of electronic devices used and harmful effect on their health

3-Is there a safeguard used by parents to protect adolescence from harmful effect of used electronic devices

\section{Subjects and Method}

\subsection{Setting}

Three secondary school classes selected randomly from Shebin el- kom Secondary Schools one for boys and other two for girls

\subsection{Sample}

Purposive sample of 126 adolescence students consists of 59 boys and 67 girls age between 16-18 yrs. whose are accepted to participate in the study were interviewed for the purpose of the research, the most digested age for using electronic

\section{Criteria for sample selection}

1-Adolescence Boys / or Girls

2-Age ranged between 16-18 Yrs.

3-Not suffered from physical impairment, or chronic disease

4-Electronic devices user

\subsection{Design}

A cross-sectional study was utilized

\subsection{Development of the tools}

Written questionnaire was designed and tested by the researcher for its validity after a through literature review and sent to a jury of three expertise two from community nursing and one professor from pediatric nursing to assess the content validity.

\subsection{Tools}

Two tools were utilized to assess the effect of electronic media on adolescence

\subsection{Tool one}

Divided into two parts

\section{Part one:}

Includes bio data about the adolescence as name, age, sex, class number, numbers of sisters, brothers, and birth orders. 


\section{Part two}

Play and Technology Questionnaire for older children The questionnaire developed by information and communication technologies (ICTs). Stella, et.al, (2007) · The questionnaire translated into Arabic and adapted by the researcher. It contains of 22 questions asking for qualitative and quantitative data as types of electronic media used by adolescence and sit of present at their homes. This questions required to be checked by yes or no The questionnaire also includes an open questions to gave adolescence the opportunity to express their own interests and priorities.

As certain technologies being present in the home?

- What kind of play do you like, both with friends and /or alone?

- What kind of technology do you have access to at home and where is it

located? - If adolescence play computer or console games, where do they play and with whom?

- Do adolescence use the Internet and for what?

- What kinds of playthings would adolescence really like to play with.

The questionnaire included reference to such technologies as tape recorders, cameras, television, musical instruments, computers, mobile phones and games devices with photo illustration for each type of electronic

\subsection{Tool Two}

An Interview Questionnaire for the use of electronic and its harmful effect on health as disturbed sleeping, lack of concentration, backache, sensitivity of the eye and other related health question. Responses were classified as non, mild, moderate or severe The use of mobile phones was assessed with the following item: 'On an average day, how long (in minutes) do you use your mobile phone for voice calls?' with the response categories 'not at all', 'Iess than 5 minutes', ' 6 to 15 minutes', '16 to 30 minutes' and 'more than 30 minutes'.

Average daily use of mobile, computer/internet, watching television/videos, playing with game consoles and listening to music was assessed using a 3-point response scale ('never', 'approximately 30 minutes', 'approximately 1 to 2 hours', 'approximately 3 to 4 hours', 'more than 4 hours'), separately for weekdays and weekend days. Average use of each electronic media was estimated as weighted means from weekdays and weekend days. An overall media index for use of electronic media was estimated by adding up time spent with computer/internet, television/videos and playing with game consoles ..Pain was stressed that from the effect of media depends on self-report and assured that to be results due to long use of electronic media only .not from any other health problems

\section{Plot Study}

In field tests the questions showed moderate to good retest reliability in 20 adolescent subjects, interview firstly before conducting the questionnaire and testing the easiest and appropriateness of the questions for the purpose of the research and do the required modifications. The pilot study was excluded from the total study

\section{Data Collection Methods}

Every adolescence was asked about his bio data, after explaining to him the purpose of the research and write his consent to participate in the study. Then the adolescence mostly filled out the questionnaires on their own. Finally, the researchers conducted the interview, which involved discussing issues about their use of TV and rules about watching TV, playing on computer or console games.. Adolescence also spoke about their use of mobile phones, the Internet and listening to music.. After that the adolescence conduct a self reporting about the degree of harmful effect of electronic as well as detect of principles of safeguard used by their parent The data was collected at the end of April 2013 and starting the administrative process from the beginning of April

\section{Consent and ethical considerations}

Permission was sought from the Principals and Boards of Management of the participating schools. Information packs were distributed to schools and included a letter explaining the purpose of the research and methods of data collection. The supervised teachers in the participating schools made the selection of class in a random basis from the number of class in the $2^{\frac{\text { nd }}{2}} \mathrm{yr}$. after the administrator giving a consent to collect data adolescence were also afforded the opportunity to give their consent to participate in the study Before each adolescence was given the questionnaire, he or she was supplied with a form to sign, agreeing to be participate in the study . ('Ethical Consent Form)',

Anonymity was afforded to all adolescence in the research and no identifying feature even the school name is not recorded in the study. Schools are not identified in the study but only their general location is referred. 


\section{Data analysis}

The collected data was categorized and analyzed by using appropriate statistical tests using SPSS version 16. Frequencies, mean, $\mathrm{x}^{2}$ and correlations were used to interpret the collected data as well as figures and graphs were used for data presentation.

VII. Results

Table (1) Distribution of biosocial characteristics of studied students and type of electronics devices used

\begin{tabular}{|c|c|c|c|}
\hline BIOSOCIAL CHARACTERISTICS & NO & $\%$ & $\mathrm{X}^{2}$ \\
\hline $\begin{array}{l}\text { Student age } \\
16 \mathrm{yrs} \\
17 \mathrm{yrs} \\
18 \mathrm{yrs}\end{array}$ & $\begin{array}{l}50 \\
36 \\
40\end{array}$ & $\begin{array}{l}39,7 \\
28.5 \\
31.7\end{array}$ & 0.000 \\
\hline Mean age & \multicolumn{2}{|c|}{$17.6 \pm 0569$} & \\
\hline $\begin{array}{l}\text { Sex } \\
\text { Boys } \\
\text { Girls }\end{array}$ & $\begin{array}{l}59 \\
67\end{array}$ & $\begin{array}{l}46.8 \\
53.2\end{array}$ & .476 \\
\hline $\begin{array}{l}\text { Birth order } \\
1^{\text {st }} \\
2^{\text {nd }} \\
3^{\text {rd }} \\
4^{\text {th }} \\
5^{\text {th }} \\
\text { others } \\
\end{array}$ & $\begin{array}{c}39 \\
46 \\
24 \\
8 \\
5 \\
4\end{array}$ & $\begin{array}{c}31.0 . \\
36.5 \\
19.0 \\
6.3 \\
4.0 \\
3.1\end{array}$ & .000 \\
\hline $\begin{array}{l}\text { Numbers of Sisters } \\
0 \\
\text { One } \\
\text { Two } \\
\text { Three } \\
\text { Four } \\
\text { Five } \\
\text { others }\end{array}$ & $\begin{array}{c}18 \\
47 \\
34 \\
15 \\
7 \\
3 \\
2\end{array}$ & $\begin{array}{c}14.3 \\
37.3 \\
27.0 \\
11.9 \\
5.6 . \\
2.4 \\
15.8\end{array}$ & .000 \\
\hline $\begin{array}{l}\text { Numbers of brothers } \\
0 \\
\text { One } \\
\text { Two } \\
\text { Three } \\
\text { others }\end{array}$ & $\begin{array}{c}17 \\
56 \\
44 \\
9 \\
0 \\
\end{array}$ & $\begin{array}{c}13.5 \\
44.4 \\
34.9 \\
7.1 \\
0.0 \\
\end{array}$ & .000 \\
\hline $\begin{array}{l}\text { Use radio } \\
\text { Yes } \\
\text { No }\end{array}$ & $\begin{array}{c}116 \\
10 \\
\end{array}$ & $\begin{array}{c}92.1 \\
7.9\end{array}$ & .000 \\
\hline $\begin{array}{l}\text { Use C.D games } \\
\text { Yes } \\
\text { No } \\
\end{array}$ & $\begin{array}{l}44 \\
82 \\
\end{array}$ & $\begin{array}{l}34.9 \\
65.1 \\
\end{array}$ & .001 \\
\hline $\begin{array}{l}\text { Use Television } \\
\text { Yes } \\
\text { No }\end{array}$ & $\begin{array}{c}124 \\
2\end{array}$ & $\begin{array}{c}98.4 \\
1.6\end{array}$ & .000 \\
\hline $\begin{array}{l}\text { Use D.V.D } \\
\text { Yes } \\
\text { No }\end{array}$ & $\begin{array}{l}28 \\
98 \\
\end{array}$ & $\begin{array}{l}22.2 \\
77.8 \\
\end{array}$ & .000 \\
\hline $\begin{array}{l}\text { Use Computer } \\
\text { Yes } \\
\text { No }\end{array}$ & $\begin{array}{c}121 \\
5\end{array}$ & $\begin{array}{c}96.0 \\
4.0\end{array}$ & .000 \\
\hline $\begin{array}{l}\text { Use Net connected Computer } \\
\text { Yes } \\
\text { No }\end{array}$ & $\begin{array}{l}74 \\
52 \\
\end{array}$ & $\begin{array}{l}58.7 \\
41.3\end{array}$ & .050 \\
\hline $\begin{array}{l}\text { Use Mobile with Camera } \\
\text { Yes } \\
\text { No } \\
\end{array}$ & $\begin{array}{c}119 \\
7 \\
\end{array}$ & $\begin{array}{c}94.4 \\
5.6 \\
\end{array}$ & .000 \\
\hline $\begin{array}{l}\text { Use Play Station } \\
\text { Yes } \\
\text { No } \\
\end{array}$ & $\begin{array}{l}45 \\
81 \\
\end{array}$ & $\begin{array}{l}35.7 \\
64.3 \\
\end{array}$ & .000 \\
\hline $\begin{array}{l}\text { Use other electronic Media } \\
\text { Yes } \\
\text { No }\end{array}$ & $\begin{array}{c}17 \\
109 \\
\end{array}$ & $\begin{array}{l}13.5 \\
86.5\end{array}$ & .000 \\
\hline
\end{tabular}

\section{$\mathrm{P}<0.0001$}

Table (1) show percentage distribution of biosocial characteristics of studied adolescence which represents that the mean age was $(17,6 \pm 0569)$, more than half of them was girls $(53,2 \%)$ and more than one third of them were 
the $2^{\text {nd }}$ birth order. The vast majority of them $(92,1 \%)$ were used radio from the mobile and watching T.V. In addition $96 \%$ of them used computer and more than half of them $(58,7 \%)$ have connected net .Also, most of them $(94,4 \%)$ used mobile phone with camera. So, there is a significance difference $\mathrm{P}<0001$ between adolescence in their uses of electronic devices

Table (2) description of adolescence used of electronic devices and duration of used

\begin{tabular}{|c|c|c|c|}
\hline STUDENTS USED OF ELECTRONIC MEDIA & No & $\%$ & $\mathbf{X}^{2}$ \\
\hline $\begin{array}{l}\text { Where do you play with electronic } \\
\text { 1- On my room } \\
\text { 2- In the hall } \\
\text { 3- In my parent room } \\
\text { 4- In my friends house } \\
\text { 5- In cybercafé }\end{array}$ & $\begin{array}{c}70 \\
26 \\
8 \\
8 \\
14 \\
\end{array}$ & $\begin{array}{c}55.5 \\
20.6 \\
6.4 \\
6.4 \\
11.1 \\
\end{array}$ & .000 \\
\hline $\begin{array}{l}\text { Do you play alone } \\
\text { Yes } \\
\text { No }\end{array}$ & $\begin{array}{l}\mathbf{6 7} \\
\mathbf{5 9}\end{array}$ & $\begin{array}{l}53.1 \\
46.9\end{array}$ & .000 \\
\hline $\begin{array}{l}\text { Like playing or watching with } \\
\text { 1- Myself } \\
\text { 2- My family } \\
\text { 3- My Friends } \\
\end{array}$ & $\begin{array}{l}46 \\
53 \\
27\end{array}$ & $\begin{array}{l}36.5 \\
42.0 \\
21.4\end{array}$ & .000 \\
\hline $\begin{array}{l}\text { Use Internet for } \\
\text { 1- School activities } \\
\text { 2- Shat } \\
\text { 3- Watching films and videos or Listen to music } \\
\text { 4-Playing Violence matches } \\
\text { 5-Send a massages }\end{array}$ & $\begin{array}{c}66 \\
30 \\
13 \\
7 \\
10\end{array}$ & $\begin{array}{l}52.3 \\
23.8 \\
10.3 \\
5.5 \\
7.9\end{array}$ & .000 \\
\hline $\begin{array}{l}\text { Type of watching on the internet when you are alone } \\
\text { 1-Films and Videos } \\
\text { 2-solcitation }\end{array}$ & $\begin{array}{c}121 \\
5\end{array}$ & $\begin{array}{c}96.0 \\
3.9 \\
\end{array}$ & .000 \\
\hline $\begin{array}{l}\text { Playing violence matches } \\
\text { 1- Yes } \\
\text { 2- No }\end{array}$ & $\begin{array}{l}52 \\
74\end{array}$ & $\begin{array}{l}41.3 \\
58.7\end{array}$ & .000 \\
\hline $\begin{array}{l}\text { Number of hrs using computer } \\
1-3 \\
4-7 \\
8-11 \\
\text { More than }\end{array}$ & $\begin{array}{c}39 \\
62 \\
22 \\
3\end{array}$ & $\begin{array}{c}30.9 \\
49.2 \\
17.4 \\
2.3 \\
\end{array}$ & .000 \\
\hline $\begin{array}{l}\text { Numbers of hrs using mobile / week } \\
1-3 \\
4-7 \\
8-11 \\
\text { More than } 11 \mathrm{hrs}\end{array}$ & $\begin{array}{c}36 \\
53 \\
31 \\
6\end{array}$ & $\begin{array}{c}28.5 \\
42,2 \\
24.6 \\
4.7\end{array}$ & .000 \\
\hline $\begin{array}{l}\text { Put mobile on bedroom during night } \\
\text { 1-Yes } \\
\text { 2-No }\end{array}$ & $\begin{array}{l}87 \\
39\end{array}$ & $\begin{array}{l}69.1 \\
30.9\end{array}$ & .000 \\
\hline $\begin{array}{l}\text { Numbers of hrs spend on face book } \\
\text { Zero } \\
1-3 \\
4-7 \\
8-11 \text { or more }\end{array}$ & $\begin{array}{l}45 \\
16 \\
41 \\
24\end{array}$ & $\begin{array}{l}35.7 \\
12.6 \\
32.5 \\
19.0\end{array}$ & .000 \\
\hline $\begin{array}{l}\text { Numbers of hrs spend on watching T.V } \\
1-3 \\
4-7 \\
8-11 \text { or more }\end{array}$ & $\begin{array}{l}22 \\
70 \\
34\end{array}$ & $\begin{array}{l}17.4 \\
55.5 \\
27.0\end{array}$ & .000 \\
\hline $\begin{array}{l}\text { Number of Minutes used mobile in day } \\
\text { Not used } \\
5-10 \text { minutes } \\
11-20 \text { minutes } \\
21-30 \text { minutes } \\
\text { More than } 30 \text { minutes }\end{array}$ & $\begin{array}{c}9 \\
37 \\
23 \\
10 \\
47\end{array}$ & $\begin{array}{c}7.1 \\
29.4 \\
18.3 \\
7.9 \\
37.3\end{array}$ & .000 \\
\hline $\begin{array}{l}\text { Parents determines Time } \\
\text { 1- Yes } \\
\text { 2- No }\end{array}$ & $\begin{array}{l}34 \\
92\end{array}$ & $\begin{array}{c}26.9 \\
73.1\end{array}$ & .000 \\
\hline Total & 126 & $\mathbf{1 0 0 . 0 0}$ & \\
\hline
\end{tabular}

Table (2) Distribution of student used of electronic devices and duration of usage. More than half of students $(55,5 \%)$ used devices inside their own room's, alone $(53,1 \%)$. While $(42 \%)$ of them like playing with media beside or with their families. Also, $(52,2 \%)$ used internet for school activities, in contrasted to $(41,3 \%)$ of them 
watching or playing violence matches, and $(3,9 \%)$ of them showing solicitation when they were alone. About half of them (40\%) using computer for 4-7 hrs/day or chatting on facebook. Also, high percentage of them $(69,1 \%)$ putting mobile inside their room's during night with more than thirty minutes used / day. However, about three quarter of them (74\%) didn't have limited time set by their parent for using electronics The difference were highly statistically significance for their used and duration of use $(\mathrm{P}<.0 .001)$

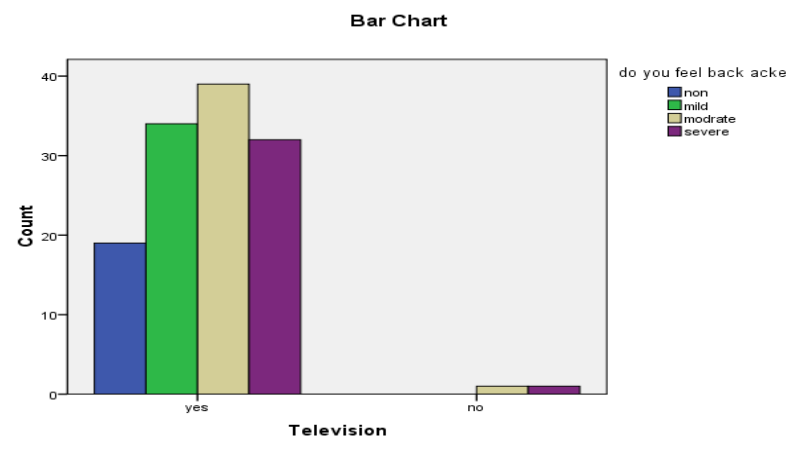

Figure (1)

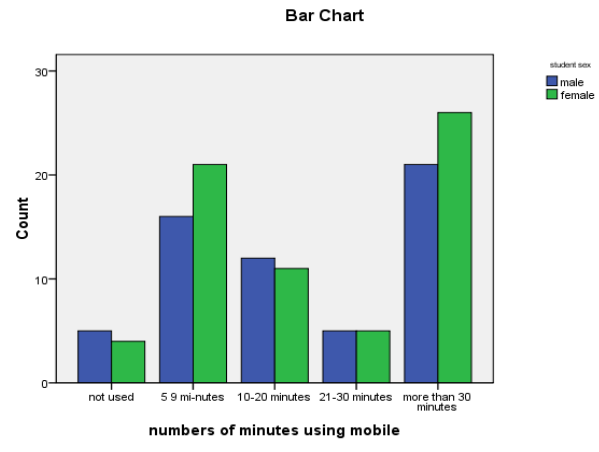

Figure (2)

Figure (1) showed distribution of students watching T.V and their feeling of moderate to severe back ache Figure (2) represented that majority of adolescent using mobile more than 30 minutes/day

Table (3) Distribution of students according to their associated symptoms related to their use of electronics

\begin{tabular}{|c|c|c|c|}
\hline $\begin{array}{l}\text { ASSOCIATED SIGNS AND SYMPTOMS RELATED } \\
\text { TO THE USED OF ELECTRONICS }\end{array}$ & $\mathrm{NO}$ & $\%$ & P.VALUE \\
\hline $\begin{array}{ll}\text { Do You have sleeping problems } \\
\text { from use of electronic } \\
1-\quad \text { Non } \\
2- & \text { Mild } \\
3- & \text { Moderate } \\
4- & \text { Severe } \\
\end{array}$ & $\begin{array}{l}21 \\
30 \\
53 \\
22\end{array}$ & $\begin{array}{l}16.7 \\
23.8 \\
42.1 \\
17.4\end{array}$ & .000 \\
\hline $\begin{array}{l}\text { Do You feel lack of concentration } \\
\text { 1-Non } \\
\text { 2-Mild } \\
\text { 3-Moderate } \\
\text { 4-Severe Non }\end{array}$ & $\begin{array}{l}18 \\
45 \\
44 \\
19\end{array}$ & $\begin{array}{l}14.2 \\
35.7 \\
34.9 \\
15.2\end{array}$ & .000 \\
\hline $\begin{array}{l}\text { Ear Ache } \\
\text { 1-Non } \\
\text { 2-Mild } \\
\text { 3-Moderate } \\
\text { 4-Severe }\end{array}$ & $\begin{array}{l}26 \\
37 \\
13 \\
50\end{array}$ & $\begin{array}{l}20.6 \\
29.4 \\
10.3 \\
39.7\end{array}$ & .000 \\
\hline $\begin{array}{l}\text { Do you feel backache } \\
\text { 1 1-Non } \\
\text { 2-Mild } \\
\text { 3-Moderate } \\
\text { 4-Severe }\end{array}$ & $\begin{array}{l}19 \\
34 \\
33 \\
40\end{array}$ & $\begin{array}{l}15.1 \\
27.0 \\
26.2 \\
31.7\end{array}$ & .057 \\
\hline $\begin{array}{l}\text { Carpal Tunnel Syndrome from using mouse } \\
\text { 1-Non } \\
\text { 2-Mild } \\
\text { 3-Moderate } \\
\text { 4-Severe }\end{array}$ & $\begin{array}{l}47 \\
20 \\
15 \\
44\end{array}$ & $\begin{array}{l}37.4 \\
15.8 \\
11.9 \\
34.9\end{array}$ & .000 \\
\hline $\begin{array}{l}\text { Do you have Itchy Eyes } \\
\text { 1-Non } \\
\text { 2-Mild } \\
\text { 3-Moderate } \\
\text { 4-Severe }\end{array}$ & $\begin{array}{l}43 \\
23 \\
19 \\
41\end{array}$ & $\begin{array}{l}34.1 \\
18.3 \\
15.1 \\
32.5\end{array}$ & .003 \\
\hline $\begin{array}{l}\text { Effect on School achievement } \\
\text { 1-Non } \\
\text { 2-Mild } \\
\text { 3-Moderate } \\
\text { 4-Severe }\end{array}$ & $\begin{array}{l}39 \\
30 \\
35 \\
22\end{array}$ & $\begin{array}{l}31.0 \\
23.8 \\
27.8 \\
17.4\end{array}$ & 164 \\
\hline
\end{tabular}

Table (3) Distribution of studied students according to their associated symptoms related to their used of electronics. About half or less of adolescence used of electronic suffering from moderate to severe form of different symptoms as sleeping problems or anxiety, beside to their feeling of lack of concentration due to their long use or lack of sleeping at night. In addition to other related complain like as earache, backache, carpal 
tunnel syndrome, itchy or sensitive eyes, Also, about one third of students effect on their school achievement So, there were a highly statistical significance difference $(\mathrm{P}<001)$ for their complaining except for their backache, itchy eyes, and school achievement $(\mathrm{P}>0.001)$

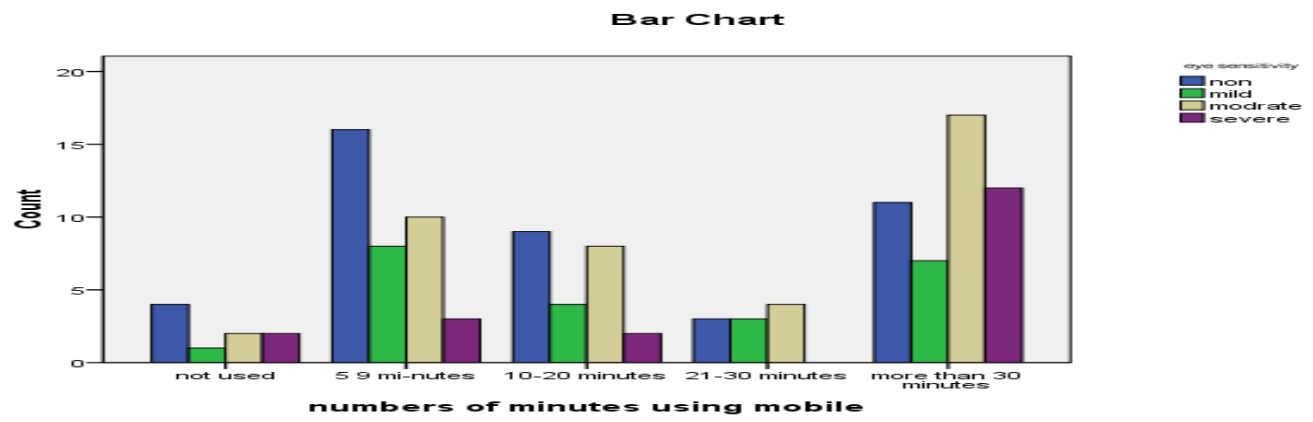

Figure (3) showed majority of students have moderate sensitivity to eyes due to massaging on mobile for more than 30 minutes//day

Table (4) Relationship between sex of students and their used of electronics

\begin{tabular}{|c|c|c|c|c|c|}
\hline \multirow[t]{3}{*}{ ADOLESCENCE USED OF ELECTRONICS } & \multicolumn{4}{|c|}{ SEX OF ADOLESCENCE } & \multirow[t]{3}{*}{$\overline{\mathrm{X}^{2}}$} \\
\hline & \multicolumn{2}{|c|}{ Boys $=59$} & \multicolumn{2}{|c|}{ GIRLS $=67$} & \\
\hline & No & $\%$ & No & $\%$ & \\
\hline $\begin{array}{l}\text { Do you use computer } \\
1 \text {-Yes } \\
2-\text { No }\end{array}$ & $\begin{array}{c}58 \\
1\end{array}$ & $\begin{array}{c}98.3 \\
1.7\end{array}$ & $\begin{array}{l}53 \\
14\end{array}$ & $\begin{array}{c}792 \\
20.8\end{array}$ & 0.002 \\
\hline $\begin{array}{l}\text { Where do you play with electronic } \\
\text { 1-On my room } \\
\text { 2-In the hall } \\
\text { 3-In my parent room } \\
\text { 4-In my friends house } \\
\text { 5-In cybercafé }\end{array}$ & $\begin{array}{c}32 \\
10 \\
5 \\
10 \\
14\end{array}$ & $\begin{array}{c}54.2 \\
16.9 \\
8.4 \\
16.9 \\
23.7\end{array}$ & $\begin{array}{l}38 \\
16 \\
3 \\
0 \\
0\end{array}$ & $\begin{array}{c}56.7 \\
23.8 \\
4.4 \\
0.0 \\
0.0\end{array}$ & .059 \\
\hline $\begin{array}{l}\text { Like playing or watching with } \\
\text { 1-Myself } \\
\text { 2-My family } \\
\text { 3-My Friends }\end{array}$ & $\begin{array}{l}19 \\
23 \\
17\end{array}$ & $\begin{array}{l}32.3 \\
38.9 \\
28.8\end{array}$ & $\begin{array}{l}27 \\
30 \\
10\end{array}$ & $\begin{array}{l}40.2 \\
44.7 \\
14.9\end{array}$ & 0.383 \\
\hline $\begin{array}{l}\text { Use Internet for } \\
\text { 4- School activities } \\
\text { 5- Chat } \\
\text { 6- Watching films and videos or Listen to music } \\
\text { 4-Playing Violence matches } \\
\text { 5-Send a massages }\end{array}$ & $\begin{array}{c}14 \\
25 \\
6 \\
4 \\
10\end{array}$ & $\begin{array}{c}23.7 \\
42.3 \\
10.1 \\
6.8 \\
16.9\end{array}$ & $\begin{array}{c}52 \\
5 \\
7 \\
3 \\
0\end{array}$ & $\begin{array}{l}77.6 \\
7.4 \\
10.4 \\
7.3 \\
0.0\end{array}$ & 0.000 \\
\hline $\begin{array}{l}\text { Type of watching on the internet when you are alone } \\
\text { 1-Films and Videos } \\
\text { 2-solcitation }\end{array}$ & $\begin{array}{c}54 \\
5\end{array}$ & $\begin{array}{c}91.5 \\
8.4\end{array}$ & $\begin{array}{c}67 \\
0\end{array}$ & $\begin{array}{c}100.0 \\
0.0\end{array}$ & 0.000 \\
\hline $\begin{array}{l}\text { Put mobile on bedroom during night } \\
1-\text { Yes } \\
2 \text {-No }\end{array}$ & $\begin{array}{l}47 \\
12\end{array}$ & $\begin{array}{l}79.6 \\
20.4\end{array}$ & $\begin{array}{l}56 \\
11\end{array}$ & $\begin{array}{l}83.6 \\
16.4\end{array}$ & 0.016 \\
\hline $\begin{array}{l}\text { Where do you put mobile during walking } \\
\text { 1- in my bag } \\
\text { 2- in my shirt pocket } \\
\text { 3- in my trousers }\end{array}$ & $\begin{array}{c}1 \\
16 \\
42\end{array}$ & $\begin{array}{l}1.7 \\
27.1 \\
71.2\end{array}$ & $\begin{array}{c}67 \\
0 \\
0\end{array}$ & $\begin{array}{c}100.0 \\
0.0 \\
0.0\end{array}$ & 0.000 \\
\hline $\begin{array}{l}\text { Do you have computer on your bed room } \\
\text { 1- Yes } \\
\text { 2- No }\end{array}$ & $\begin{array}{l}34 \\
25\end{array}$ & $\begin{array}{l}57.6 \\
42.3\end{array}$ & $\begin{array}{l}35 \\
32\end{array}$ & $\begin{array}{l}52.3 \\
47.7\end{array}$ & 0.004 \\
\hline $\begin{array}{l}\text { Violence increased through watching action films } \\
1-\text { Yes } \\
2-\text { No }\end{array}$ & $\begin{array}{l}41 \\
18\end{array}$ & $\begin{array}{l}69.5 \\
30.5\end{array}$ & $\begin{array}{l}11 \\
56\end{array}$ & $\begin{array}{l}14.5 \\
83.5\end{array}$ & 0152 \\
\hline
\end{tabular}

Table ( 4) Relationship between sex of adolescence and their used of electronics. There were a significance difference $(\mathrm{P}<0.005)$ between boys and girls in relation to their used of electronic devices in regards to their using computer, and internet, type of watching, site of carrying the mobile during walking, or setting of computer on their homes. On the other hand, there were in significance difference $(\mathrm{P}>0.001)$ relationship between both sex regarding to their place of playing electronic, play or watching computer with, and increase violence from their watching action films 
Table (5) Relationship between students sex and their time determined of using electronic devices

\begin{tabular}{|c|c|c|c|c|c|}
\hline \multirow{3}{*}{$\begin{array}{l}\text { DURATION OF TIME USED ELECTRONIC BY } \\
\text { ADOLESCENCE }\end{array}$} & \multicolumn{4}{|c|}{ SEX OF THE ADOLESCENCE } & \multirow[t]{3}{*}{$X^{2}$} \\
\hline & \multicolumn{2}{|c|}{ BOYS $=59$} & \multicolumn{2}{|c|}{ GIRLS=67 } & \\
\hline & No & $\%$ & No & $\%$ & \\
\hline $\begin{array}{l}\text { Number of hrs using computer } \\
1-3 \\
4-7 \\
8-11 \\
\text { More than }\end{array}$ & $\begin{array}{c}10 \\
29 \\
10 \\
1\end{array}$ & $\begin{array}{c}32.2 \\
49.1 \\
16.9 \\
1.7\end{array}$ & $\begin{array}{c}20 \\
34 \\
12 \\
1\end{array}$ & $\begin{array}{c}29.8 \\
50.7 \\
17.9 \\
1.4\end{array}$ & .152 \\
\hline $\begin{array}{l}\text { Numbers of hrs using mobile / week } \\
1-3 \\
4-7 \\
8-11 \\
\text { More than } 11 \mathrm{hrs} \\
\end{array}$ & $\begin{array}{c}6 \\
30 \\
17 \\
6 \\
\end{array}$ & $\begin{array}{l}10.1 \\
50.8 \\
28.8 \\
10.1 \\
\end{array}$ & $\begin{array}{c}33 \\
32 \\
5 \\
0 \\
\end{array}$ & $\begin{array}{c}49.2 \\
42.1 \\
7.4 \\
0.0 \\
\end{array}$ & .0 .000 \\
\hline $\begin{array}{l}\text { Numbers of hrs spend on watching T.V } \\
1-3 \\
4-7 \\
8-11 \\
\text { More than } 11 \mathrm{hrs} \\
\end{array}$ & $\begin{array}{c}6 \\
32 \\
20 \\
1\end{array}$ & $\begin{array}{l}10.1 \\
54.2 \\
33.8 \\
1 . .7 \\
\end{array}$ & $\begin{array}{c}16 \\
39 \\
11 \\
1 \\
\end{array}$ & $\begin{array}{c}23.6 \\
58.2 \\
16.4 \\
1.4 \\
\end{array}$ & 0.37 \\
\hline $\begin{array}{l}\text { Number of Minutes used mobile in day } \\
\text { Not used } \\
5-10 \text { minutes } \\
11-20 \text { minutes } \\
21-30 \text { minutes } \\
\text { More than } 30 \text { minutes }\end{array}$ & $\begin{array}{c}5 \\
16 \\
12 \\
5 \\
21\end{array}$ & $\begin{array}{c}8.4 \\
27.1 \\
20.3 \\
8.4 \\
35.5 \\
\end{array}$ & $\begin{array}{c}4 \\
21 \\
11 \\
5 \\
26\end{array}$ & $\begin{array}{c}5.2 \\
31.3 \\
16.4 \\
7.4 \\
38.8 \\
\end{array}$ & .931 \\
\hline $\begin{array}{l}\text { Numbers of hrs spend on face book } \\
\text { Zero } \\
1-3 \\
4-7 \\
8-11 \text { or more } \\
\end{array}$ & $\begin{array}{c}15 \\
23 \\
20 \\
1\end{array}$ & $\begin{array}{c}25.4 \\
38.9 \\
33.8 \\
1.6\end{array}$ & $\begin{array}{c}46 \\
18 \\
4 \\
0\end{array}$ & $\begin{array}{c}68.6 \\
26.8 \\
5.9 \\
0.0\end{array}$ & 0.000 \\
\hline $\begin{array}{ll}\text { Do you } & \text { watching action matches } \\
1- & \text { yes } \\
2- & \text { No } \\
\end{array}$ & $\begin{array}{l}25 \\
34 \\
\end{array}$ & $\begin{array}{l}42.3 \\
57.6 \\
\end{array}$ & $\begin{array}{c}8 \\
58 \\
\end{array}$ & $\begin{array}{l}11.9 \\
85.8\end{array}$ & 0.000 \\
\hline $\begin{array}{l}\text { Do you chat with your friends } \\
\text { 1-yes } \\
\text { 2-No }\end{array}$ & $\begin{array}{l}40 \\
19 \\
\end{array}$ & $\begin{array}{l}57.7 \\
32.2 \\
\end{array}$ & $\begin{array}{l}13 \\
54 \\
\end{array}$ & $\begin{array}{l}19.4 \\
80.5 \\
\end{array}$ & 0.000 \\
\hline $\begin{array}{l}\text { Parents determines Time } \\
\text { 1-Yes } \\
\text { 2- No }\end{array}$ & $\begin{array}{l}14 \\
20 \\
\end{array}$ & $\begin{array}{l}23.7 \\
33.8 \\
\end{array}$ & $\begin{array}{l}18 \\
47 \\
\end{array}$ & $\begin{array}{l}26.8 \\
70.1 \\
\end{array}$ & .712 \\
\hline $\begin{array}{l}\text { Parent Determine content of electronic used } \\
\text { Yes } \\
\text { No }\end{array}$ & $\begin{array}{c}0 \\
59 \\
\end{array}$ & $\begin{array}{c}0.00 \\
100.00 \\
\end{array}$ & $\begin{array}{l}10 \\
57 \\
\end{array}$ & $\begin{array}{l}14.9 \\
85.1 \\
\end{array}$ & 0.000 \\
\hline
\end{tabular}

Table (5) Relationship between students sex and their duration and using electronic devices. There were a significance difference $(\mathrm{P}<0.001)$ between both sex ( boys, and girls) in their duration of uses of electronic devices/ day, number of hrs. using mobile/ week, number of hrs chatting on the facebook, or watching action matches as well as parent determination of electronic devices content. In contrast to in significance difference $(\mathrm{P}>0.001)$ between both sex regarding to their used of computer, number of hrs. spending on watching T.V, or their time determined by their parents for their used

Table (6) Relationship between student sex and associated signs and symptoms from their used of electronic devices

\begin{tabular}{|c|c|c|c|c|c|}
\hline \multirow{3}{*}{$\begin{array}{l}\text { ASSOCIATED SIGNS AND SYMPTOMS RELATED } \\
\text { TO THE USED OF ELECTRONICS }\end{array}$} & \multicolumn{4}{|c|}{ SEX OF ADOLESCENCE } & \multirow[t]{3}{*}{$\mathrm{X}^{2}$} \\
\hline & \multicolumn{2}{|c|}{ Boys $=59$} & \multicolumn{2}{|c|}{ Girls $=67$} & \\
\hline & No & $\%$ & No & $\%$ & \\
\hline $\begin{array}{l}\text { Do You have sleeping problems } \\
\text { 1-Non } \\
\text { 2-Mild } \\
\text { 3-Moderate } \\
\text { 4-Severe }\end{array}$ & $\begin{array}{l}15 \\
13 \\
18 \\
13 \\
\end{array}$ & $\begin{array}{l}25.4 \\
22.0 \\
30.5 \\
22.0 \\
\end{array}$ & $\begin{array}{c}6 \\
17 \\
35 \\
9 \\
\end{array}$ & $\begin{array}{c}8.9 \\
25.3 \\
52.2 \\
13.4 \\
\end{array}$ & $0 . .018$ \\
\hline $\begin{array}{l}\text { Do You feel lack of concentration } \\
\text { 1 1-Non } \\
\text { 2-Mild } \\
\text { 3-Moderate } \\
\text { 4-Severe }\end{array}$ & $\begin{array}{l}11 \\
16 \\
22 \\
10\end{array}$ & $\begin{array}{l}16.6 \\
27.2 \\
37.2 \\
16.9 \\
\end{array}$ & $\begin{array}{c}7 \\
28 \\
22 \\
10 \\
\end{array}$ & $\begin{array}{l}11.8 \\
41.7 \\
32.8 \\
14.9 \\
\end{array}$ & .371 \\
\hline $\begin{array}{l}\text { Ear Ache } \\
\text { 1-Non } \\
\text { 2-Mild } \\
\text { 3-Moderate }\end{array}$ & $\begin{array}{l}18 \\
16 \\
18 \\
\end{array}$ & $\begin{array}{l}30.5 \\
27.1 \\
30.5 \\
\end{array}$ & $\begin{array}{c}8 \\
21 \\
32 \\
\end{array}$ & $\begin{array}{l}11.9 \\
31.3 \\
47.7 \\
\end{array}$ & .045 \\
\hline
\end{tabular}




\begin{tabular}{||l|c|c|c|c|c||}
\hline 4-Severe & 7 & 11.8 & 6 & 8.9 & \\
\hline Do you feel backache & & & & & \\
1-Non & 10 & 16.9 & 9 & 13.4 & \\
2-Mild & 13 & 22.0 & 21 & 31.3 & .093 \\
3-Moderate & 15 & 25.4 & 25 & 37.3 & \\
4-Severe & 21 & 35.5 & 12 & 17.9 & \\
\hline Carpal Tunnel Syndrome & & & & & \\
1-Non & 29 & 49.1 & 18 & 26.8 & \\
2-Mild & 12 & 20.3 & 8 & 11.9 & 0.000 \\
3-Moderate & 9 & 15.2 & 35 & 52.3 & \\
4-Severe & 9 & 15.2 & 6 & 8.9 & \\
\hline Do you have Itchy Eyes & 27 & & & & \\
1-Non & 9 & 45.7 & 16 & 23.6 & \\
2-Mild & 11 & 15.2 & 14 & 20.8 & .004 \\
3-Moderate & 12 & 20.3 & 30 & 44.7 & \\
4-Severe & & & 7 & 10.4 & \\
\hline Effect on School achievement & 16 & 27.1 & 23 & 34.2 & \\
1-Non & 11 & 18.6 & 19 & 28.3 & .003 \\
2-Mild & 11 & 18.6 & 24 & 35.8 & \\
3-Moderate & 21 & 35.5 & 1 & 1.4 & \\
4-Severe & & & \\
\hline
\end{tabular}

Table (6) Relationship between student sex and associated signs and symptoms from their used of electronic devices. There were no statistical significance difference $(\mathrm{P}>0.05)$ between both sexes (boys \& girls) from associated signs and symptoms from their used of electronic devices on their sleeping, concentration, ear, or backache. In contrast to a statistical significance difference $(\mathrm{P}<0.05)$ from their electronic devices use on hands, eyes and school achievement

\section{Table (7) Comparison between different electronic devices commonly used and their effect of} health of adolescence

Table (7) Comparison between different electronic devices and their effect on health of adolescence. About half or more than half percent of adolescence suffer from moderate sleeping problem due to their

\begin{tabular}{|c|c|c|c|c|c|c|c|c|}
\hline \multirow{2}{*}{$\begin{array}{l}\text { ASSOCIATED SYMPTOMS RELATED } \\
\text { TO THE USED OF ELECTRONICS }\end{array}$} & \multicolumn{2}{|c|}{ COMPUTER } & \multicolumn{2}{|c|}{ T.V } & \multicolumn{2}{|c|}{ "MOBILE } & \multicolumn{2}{|c|}{ C.D GAMES } \\
\hline & $\mathrm{No}=126$ & $\%$ & $\mathrm{No}=126$ & $\%$ & $\mathrm{No}=126$ & $\%$ & $\mathrm{No}=126$ & $\%$ \\
\hline $\begin{array}{l}\text { Do You have sleeping problems } \\
\text { 1-Non } \\
\text { 2-Mild } \\
\text { 3-Moderate } \\
\text { 4-Severe }\end{array}$ & $\begin{array}{l}20 \\
30 \\
50 \\
26\end{array}$ & $\begin{array}{l}15.8 \\
23.8 \\
39.6 \\
20.6\end{array}$ & $\begin{array}{l}21 \\
29 \\
55 \\
21\end{array}$ & $\begin{array}{l}16.6 \\
23.0 \\
43.6 \\
16.6\end{array}$ & $\begin{array}{l}20 \\
30 \\
48 \\
28\end{array}$ & $\begin{array}{l}15.8 \\
23.8 \\
38.0 \\
22.2\end{array}$ & $\begin{array}{c}40 \\
20 \\
65 \\
1\end{array}$ & $\begin{array}{l}31.7 \\
15.8 \\
51.5 \\
0.08\end{array}$ \\
\hline $\begin{array}{l}\text { Do You feel lack of concentration } \\
\text { 1 1-Non } \\
\text { 2-Mild } \\
\text { 3-Moderate } \\
\text { 4-Severe }\end{array}$ & $\begin{array}{l}18 \\
44 \\
42 \\
22\end{array}$ & $\begin{array}{l}14.2 \\
34.9 \\
33.3 \\
17.3\end{array}$ & $\begin{array}{l}18 \\
44 \\
46 \\
18\end{array}$ & $\begin{array}{l}14.5 \\
35.4 \\
36.5 \\
14.5\end{array}$ & $\begin{array}{l}17 \\
43 \\
46 \\
20\end{array}$ & $\begin{array}{l}13.4 \\
34.1 \\
36.5 \\
15.8\end{array}$ & $\begin{array}{c}10 \\
7 \\
50 \\
40\end{array}$ & $\begin{array}{l}22.7 \\
15.9 \\
39.6 \\
31.7\end{array}$ \\
\hline $\begin{array}{l}\text { Do you feel ear ache } \\
\text { 1-Non } \\
\text { 2-Mild } \\
\text { 3-Moderate } \\
\text { 4-Severe }\end{array}$ & $\begin{array}{l}0 \\
0 \\
0 \\
0\end{array}$ & $\begin{array}{l}0.0 \\
0.0 \\
0.0 \\
0.0\end{array}$ & $\begin{array}{l}48 \\
37 \\
26 \\
15\end{array}$ & $\begin{array}{l}38.0 \\
29.3 \\
20.6 \\
11.9\end{array}$ & $\begin{array}{l}25 \\
35 \\
14 \\
52\end{array}$ & $\begin{array}{l}19.8 \\
27.7 \\
11.1 \\
41.2\end{array}$ & $\begin{array}{c}69 \\
32 \\
21 \\
4\end{array}$ & $\begin{array}{c}54.7 \\
25.3 \\
16.6 \\
3.1\end{array}$ \\
\hline $\begin{array}{l}\text { Do you feel backache } \\
\text { 1-Non } \\
\text { 2-Mild } \\
\text { 3-Moderate } \\
\text { 4-Severe }\end{array}$ & $\begin{array}{l}19 \\
20 \\
31 \\
56\end{array}$ & $\begin{array}{l}15.2 \\
15.8 \\
24.6 \\
44.4\end{array}$ & $\begin{array}{l}19 \\
34 \\
32 \\
41\end{array}$ & $\begin{array}{l}15.2 \\
26.9 \\
25.3 \\
32.5\end{array}$ & $\begin{array}{l}35 \\
32 \\
37 \\
22\end{array}$ & $\begin{array}{l}27.7 \\
25.3 \\
29.3 \\
17.4\end{array}$ & $\begin{array}{c}7 \\
11 \\
53 \\
55\end{array}$ & $\begin{array}{c}5.5 \\
8.7 \\
42.0 \\
43.6\end{array}$ \\
\hline $\begin{array}{l}\text { Do you feel Carpal Tunnel Syndrome } \\
\text { 1-Non } \\
\text { 2-Mild } \\
\text { 3-Moderate } \\
\text { 4-Severe }\end{array}$ & $\begin{array}{l}44 \\
20 \\
15 \\
47\end{array}$ & $\begin{array}{l}43.9 \\
15.8 \\
11.9 \\
37.3\end{array}$ & $\begin{array}{c}126 \\
0 \\
0 \\
0\end{array}$ & $\begin{array}{c}100.0 \\
0.00 \\
0.00 \\
0.00\end{array}$ & $\begin{array}{l}20 \\
18 \\
41 \\
45\end{array}$ & $\begin{array}{l}15.8 \\
14.2 \\
32.5 \\
35.7\end{array}$ & $\begin{array}{l}11 \\
19 \\
63 \\
33\end{array}$ & $\begin{array}{c}8.7 \\
15.7 \\
50.0 \\
26.1\end{array}$ \\
\hline $\begin{array}{l}\text { Do you feel Itchy Eyes } \\
\text { 1-Non } \\
\text { 2-Mild } \\
\text { 3-Moderate } \\
\text { 4-Severe }\end{array}$ & $\begin{array}{l}22 \\
38 \\
23 \\
43\end{array}$ & $\begin{array}{l}17.4 \\
31.1 \\
18.2 \\
34.1\end{array}$ & $\begin{array}{l}43 \\
22 \\
20 \\
41\end{array}$ & $\begin{array}{l}34.1 \\
17.3 \\
15.8 \\
32.5\end{array}$ & $\begin{array}{l}40 \\
23 \\
43 \\
19\end{array}$ & $\begin{array}{l}31.7 \\
18.2 \\
34.1 \\
15.7\end{array}$ & $\begin{array}{l}16 \\
21 \\
70 \\
19\end{array}$ & $\begin{array}{l}12.6 \\
16.6 \\
55.5 \\
15.0\end{array}$ \\
\hline $\begin{array}{l}\text { Effect on School achievement } \\
\text { 1-Non } \\
\text { 2-Mild } \\
\text { 3-Moderate } \\
\text { 4-Severe }\end{array}$ & $\begin{array}{l}36 \\
29 \\
33 \\
28\end{array}$ & $\begin{array}{l}28.5 \\
23.1 \\
26.1 \\
22.2\end{array}$ & $\begin{array}{l}39 \\
30 \\
33 \\
24\end{array}$ & $\begin{array}{l}30.9 \\
23.8 \\
26.1 \\
19.2\end{array}$ & $\begin{array}{l}37 \\
29 \\
31 \\
29\end{array}$ & $\begin{array}{l}29.3 \\
23.1 \\
24.6 \\
23.0\end{array}$ & $\begin{array}{l}15 \\
13 \\
87 \\
11\end{array}$ & $\begin{array}{c}11.7 \\
10.2 \\
69.4 \\
8.7\end{array}$ \\
\hline
\end{tabular}

watching T.V and using C.D games which leads to lack of concentration with different degree. Using mobile is the most cause of moderate form of earache for more than (40\%) of adolescence. Also, using Computer and C.D games are the most cause of moderate to severe backache, carpal tunnel syndrome and itchy eyes as well as effect on their school achievement for most of adolescence.(69\%) . 
Bar Chart

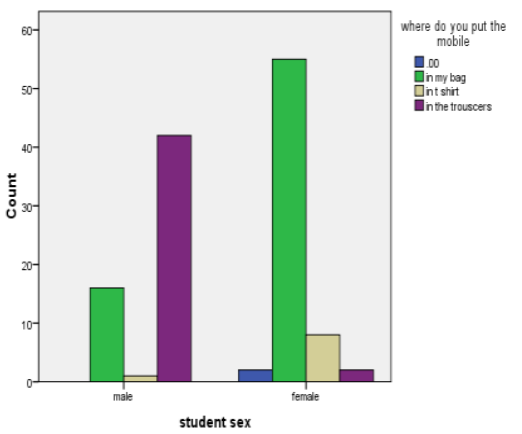

Figure (4)
Bar Chart

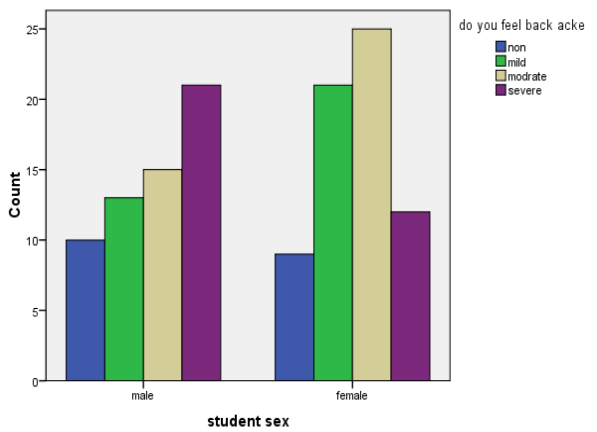

Figure (5)

Figure (4) represents site of carrying mobile during walking. Majority of boys put it in trousers and girls put it in their bags

Figure (5) relationship between adolescence sex and their backache which described as severs among girls than boys

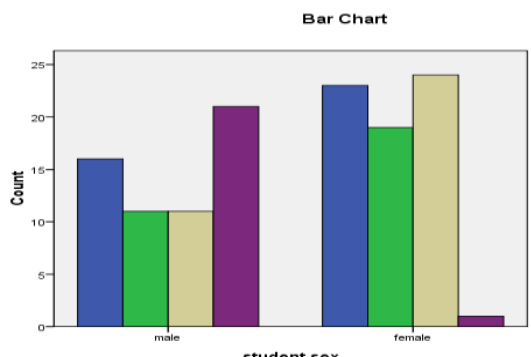

Figure (6)

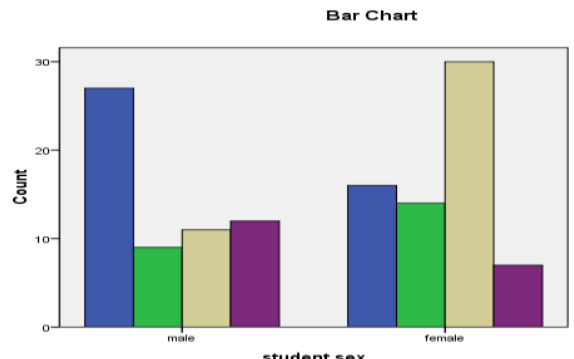

Figure (7)

Figure (6) represent relationship between sex of students and effect of electronic on their school achievement which showed that severe effect on boys and moderate effect on girls

Figure (7) showed relationship between student sex and sensitivity of their eyes which represents that majority of girls suffer from moderate forms of eye sensitivity while majority of boys didn't suffer from it.
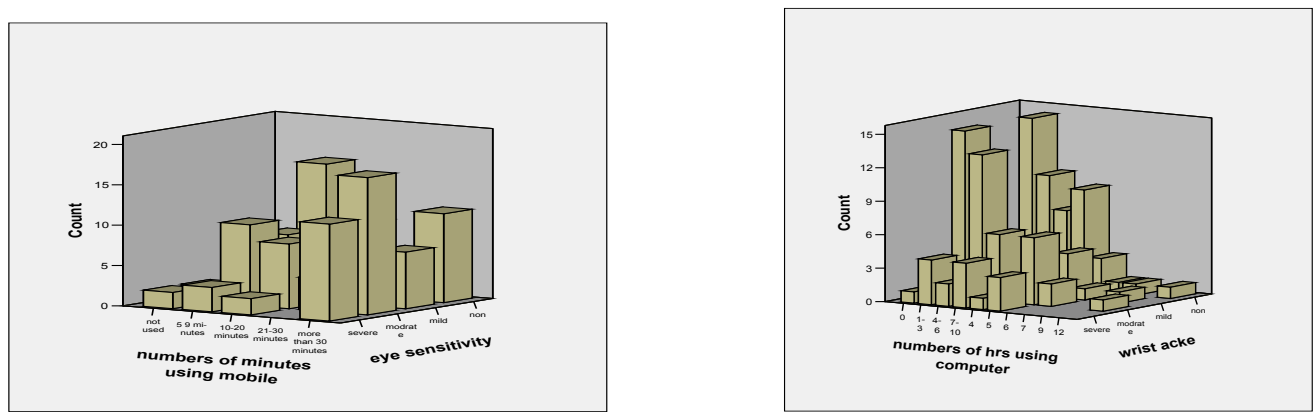

Figure (8) relationship between numbers of minutes adolescent use mobile with their eyes sensitivity represent correlation with mild forms of itchy eyes

Figure (9) represent relationship between moderate effect on wrist of adolescent due to their long time use of computer 

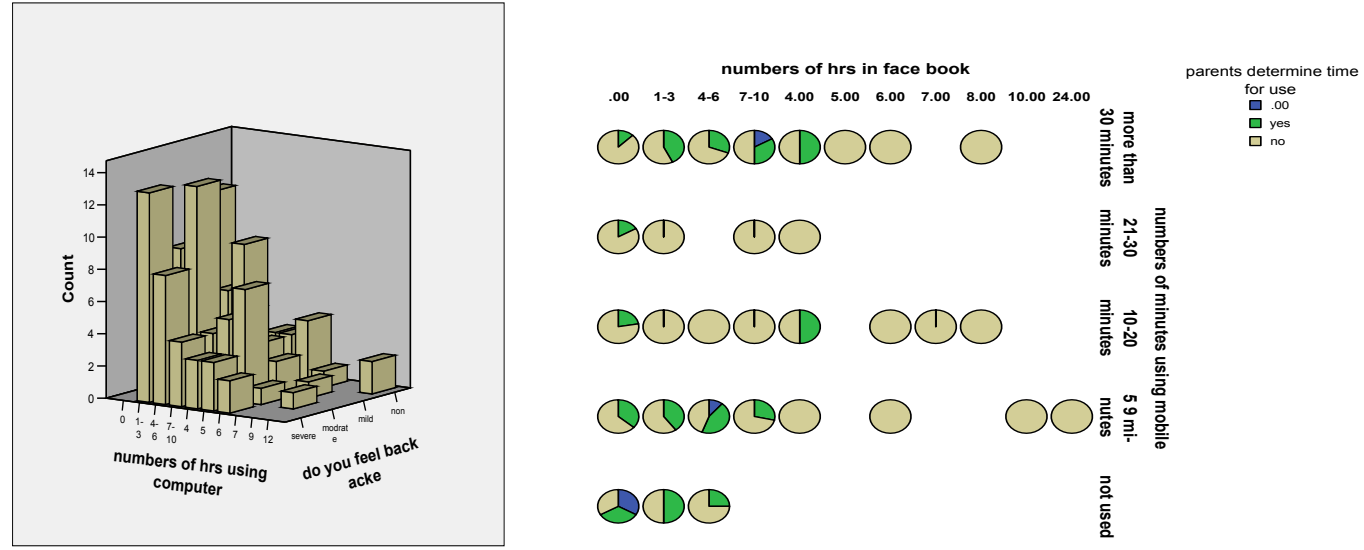

Figure (10) represent relationship between number of hrs. students using computer and their sensation of moderate to severe backache.

Figure (11) represent numbers of hrs. spend in facebook, number of minutes using mobile and parent determinate time for use which represents that majority of parents didn't determine time for electronic use

\section{Discussion}

The presence and intensity of electronic devices influences on everyday life of adolescence are increasingly recognized as an important part of the social and educational ecology of youth. So, these influences have become more visible and volatile in recent decades. Research that explores the level and effects of these devices influences calls for measurements of the quantity and character of exposure to a variety of potentially overlapping electronic sources, needs for analysis of its effect on their health, social, and educational achievement.

This study explore the types of electronic media used by adolescence as well as the degree of health effect related to their electronic used. Because of the availability of different types of electronic devices access to approximately all adolescence to provide them with different learning, educational and internet experience, All those media devices are replaces time spent interacting with caregivers and other friends. Also, social interaction is critical to a adolescence healthy development This results were consistent with (Grabioandowski,2012) who mentioned that electronic media is a daily presence in the live of most of adolescence as television, videogames on console, computers and mobile which are widely used by them. So, how much those media affect on adolescence health and development depends on how long they still consuming those electronics. For this reason they should be carefully monitored and kept with in safe time limits when viewing or playing on any of them. The mean age for those adolescence was $(17,6+056)$ and the number of girls were exceeded than boys $(53,3 \%, 46,8 \%$ respectively) with significance difference $(\mathrm{P}<0.05)$ in their number of hrs using mobile, chatting in facebook, watching action matches, contrasted with in significance difference( $\mathrm{P}>0.05$ ) between them in their using computer, watching T.V or number of minutes talking in mobile. Beverly, et.al (2012) examine how both electronic media used and exposure vary with demographic factors such as age race and he note that exposure to electronic begins early, and increases until children begin school, drops off briefly, then climbs again to peak at almost eight hours daily among eleven- and twelve-year-olds. Television and video exposure is particularly high among youth. This behaviors may be due to the pattern of development of this stage when the adolescences mainly thought by their mind.

With the exploration of the sample, more than half of adolescence watch or play with an electronic devices alone on their rooms which expose them to do un ethical behavior or lack of self-control, Also, because they are often played alone, they could also foster social isolation.

In addition to, there were some of them watching to solicitations. By comparing the study conducted by Chang and Hongisk (2005) reported that quarter of the respondents $(\mathrm{n}=150$, ages 10-17 yrs) receiving unwanted exposure to sexual materials, and less than quarter received a sexual solicitation while online. To reduce this behavior, parents are encouraged to placing computers in a central location of the house rather than putting them in adolescence room where that it always possible to be aware of the type of online activities they are engaged in . In addition, when adolescence showed sexual issues on T.V with parent they should discussed it to be less likely to occur later on when they are alone. This is include $1^{\text {st }}$ and $2^{\text {nd }}$ level of safeguard which need recognizing the potential indicator of child maltreatment, which including parent to look for possible harm and knowing who to contact and seek advice and taking appropriate action and reporting concerns,

According to (Internet Advisory Board, 2001) reported that most of parents of teenager didn't realize that their teenagers have visited inappropriate web sites. So, parent should have control over adolescence internet usage. Chang and Hongisk (2005) implies the importance of identifying other significant factors for 
electronic devices use such as family relationship, interaction and control as an important antecedent to safe guarding against negative impact of electronic use. For those reasons, parents should checking the settings for any malicious social network sites

More than half of a adolescence like watching or playing alone and spending an average of 4-7 hrs watching or playing with electronic devices and like to play violence matches. Furthermore, TV often promotes violent acts as a fun and effective way to get what they want, without consequences.

In addition, watching T.V affecting adolescence sleep. Researchers report that when adolescence spend time watching television and playing video games, it arouses, or awakens, their brains; that kind of arousal makes it that much harder for those to relax and fall asleep quickly.

Researchers also indicated that the backlighting from TV and gaming system screens can affect adolescence's circadian rhythms. which are responsible for telling us when it's time to fall asleep and time to wake up. So, anything that throws off our circadian rhythms changing sleeping pattern, So, healthcare providers confirm that over time, chronic sleep deprivation takes its toll. .This chronic sleep deprivation spells big problems for adolescence. (The Baby Sleep Site 2013)

( National Academy of Science,2012) stated that the number of hours young people spend interacting in some way with media, as well as the range and capabilities of the many electronic devices and activities that could be considered media experiences, have increased to an extent far beyond the imagining of today's. In addition (Robson,et,al, 2001) study the association between violence in media and violence behavior which result from lack of supervision. So, parent should advice to limit showing of such matches. Also, adolescence should be encourage to evaluate what they are watching or visited in the websites which promote love, affirmation and promote intimate relationship with parents.

In relation to the use of adolescence to mobile, the vast majority of them putting their mobile at night on their rooms, and more than forty percent spending 4-7 hrs /week talking with friend with more than thirty minutes in average day which affect those adolescence on their sleeping from the effect of meltonine production (Bluck, 2007). In addition more than thirty percent of those adolescence suffer from moderate lack of concentration, and severe backache. (Lioyed \& Brodie,2000) mentioned that adolescence uses computer and electronic games may adopt the kinds of sustained and awaked postures that associated with musculoskeletal disorders, and the physical demand of extensive use which may lead to a wide rang of adverse effect on their development includes visual, neurological and physical changes. So, specialist in child health, pediatricians and nurses are potentially have the power to address this issues which raised to the public to eliminate those health hazards to be effect on healthy development of adolescence. This include $3^{\text {rd }}$ level of safeguard which contributes to assessing, planning, interviewing , and evaluating problems, then they should drew on child and family focused clinical and professional knowledge to identify signs of physical or sexual abuse.

Pattern of sleep problems have also been associated with TV viewing behavior among more than forty percent of adolescence suffering from interrupted sleep likely due to presence of TV or computer on their bed room. Clinicians should stress that parents should understand that spending minute discussing hazards of electronic media with adolescence may be as important as explaining the importance of a bicycle helmet for their safety. American life project (2008) found that most of teenagers go on line and the majority uses social network site, girls play an average of about $(5.5 \mathrm{hr} /$ week )and boys average spend (13 hrs / week) playing games which is not limited to adolescence boy. In addition video game habits are correlated with risk factors for health and poor academic performance for boys more than girls, this may be due to life pattern of boys than girls. Like those adolescence which they moderately effected on their school performance by diffidence use electronic media .In addition to, (Sara et.al, 2011) found more link between media exposure and poor school achievement which causes attention deficit disorder. Also small link showed between heavy TV viewing and non clinical attention deficit

Moreover, more than thirty percent of adolescence suffer from severe carpal tunnel syndrome and itchy eyes. Grabianowski (2012) stated that long use of computer, mobile, or T.V can strain eyes which leads to worsen the existing eye condition and difficulty in focusing, So, it is important to rest eyes while working in computer or watching T.V and looking at more long distance object. Smith et, al: $\mathbf{( 2 0 0 9 , )}$ stated that over the long term of use, computer can cause physical damage, using a mouse and keyboard for many hrs/a day which can lead to repetitive stress injuries. For those reason its important to take frequent break from long use of different electronic use. In addition to back problems which are common among adolescence who spend a lot of time which is commonly ranged from 4-7 hrs. Moreover, longtime used of electronic devices deprive those adolescence from sleeping which lead them to drowsiness, difficult in concentration and inhibition of immune system as well as poor physical condition and even obesity (Grabindowski,2012). So, nurses should be capable to fulfill their duties, and examine link between use of media and its harmful effect (Woodord \&Gridina,2000) This include levels 4 and 5 of safeguard which they should work in team and partner in other agencies training and teaching for staff for case management and analyze its causes to plan appropriate strategies to ensure adherence to legislation and policy for quality management. 
More than thirty percent of adolescence suffer from moderate ear ache among both sexes which result from their long use of mobile or musculoskeletal problems results from texting massage ( Sara, et.al, 2011) reporting that symptoms associated with using of mobile phone most commonly includes headaches, earache warmth sensation, perceived difficult concentration and fatigue.

This is may be due to the Specific Absorption Rate (SAR) used for declaration of mobile phone safety, which equal to $2 \mathrm{w} / \mathrm{kg}$ average over ten gram of brain tissue, can not be viewed as sufficiently scientifically ground, which doesn't guarantee protection of brain tissues from this rays which affect on adolescence health. So, its important to protect adolescence from harmful health effect through multidisciplinary team .including expertise in different electronic rays adherence to guidelines for electronic devices use which influenced by parental and adolescence education including level $6^{\text {th }}$ of safeguard encompassing legal proceeding of specialty area to government to notifying the nations about standard and national strategies for safe use . In addition to, written consent about the use of each electronic devices should be clearly defined by their industries to safeguard users from harmful effect. ( Sara, et.al, 2011)

\section{Conclusion}

Electronic devices expose adolescence to social, behavior or moderate to severe hazards of backache, carpal tunnel syndrome, itchy eyes, and sleeping problems which lead to lack of concentration, and affection of their school performance. So, its important to entertain and educate adolescence and parents from each electronic devices and considering that your health is the first. In addition Parent, nurses, physicians should fulfill their roles to safeguard adolescence from any harmful effect during their use of electronic using guided book for every device

\section{Recommendations}

1- Written instruction about the use of each electronic media for adolescence should be clearly defined by industries to safeguard them from harmful effect

2- Awareness campaign for parent should be held in mass media about the appropriate use of media to prevent social, behavioral and physical hazards

3-Continouing medical and nursing educational programs should be planned at clinical level to guide parent about the appropriate use of electronic media and aware them about physical, educational and behavior hazards. 4- Develop the competences of all health staff to recognize adolescence maltreatment and take effective action appropriate to their role

\subsection{Recommendation for adolescence}

1- Visits the optician and general practitioner for regularly check up and make sure to tell them if they are frequently electronic media users

\subsection{Recommendation for research}

Up on requested research, the capabilities and mitigation possibilities of particular electronic devices need coordination with its manufactures and target group used to provide guidance with best practice for conducting with each device.

\section{References}

[1] American Life Project (2008).Research Center's Internet, Washington,DC, United States1615 L Street NW

[2] Bandura N. (1994) .Social cognitive theory of mass communication. In: Bryant J, Zillmann D eds. Media Effects: Advances in Theory and Research. Hillsdale, NJ:, Lawrance 61-90

[3] Beverly A. Bondad- B, Ronald E \& Katy E. (2012). Influences on TV Viewing and Online User-shared Video Use: Demographics, Generations, Contextual Age, Media Use, Motivations, and Audience Activity Journal of Broadcasting \& Electronic Media Volume 56, Issue 4, pages 471-493

[4] Bluck H,(2007). The Association between Use of Mobile Phones after Lights Out and Sleep Disturbances among Japanese Adolescents: A Nationwide Cross-Sectional Survey Sleep. 2011 August 1; 34(8): 1013-1020.

[5] Brad J. and. Rowell H, (2001).Effects of Televised Violence on Aggression," in The Handbook of Children and Media, edited by Dorothy and Jerome Singer (Thousand Oaks, Calif.: Sage Publications, pp. 223-54.

[6] Chang-H, and Hongsik J. (2005). Children's exposure to negative Internet content: effects of family context

[7] Ellen A.,(2000). The Future of Children and Computer Technology Vol. 10•No. (2) Fall/Winter 2000

[8] Gentile, D.. Lynch, P., Linder, J. and Walsh, D. (2004). The effects of violent video game habits on adolescent hostility, aggressive behaviors, and school performance. Journal of Adolescence, 27, 5-22.

[9] Gentile D, and Walsh D. (2002;). A normative study of family media habits. Appl Dev Psychol. 23:157-178.

[10] Grabianowski E,(2012). How Computer addiction Works Kansas State University. 1998-2012 How Stuff Works, Inc

[11] ( Information Center, Egyptian Journal of El Ahram Friday ,2012)

[12] Internet Advisory Board. (2001). Research of Internet downside issues. Retrieved September 4, 2004, from http://www.iab.ie/Publications/Research 
[13] Jeanne B, and Elisabeth H,(2008). Children and Electronic media Vol. 18 / NO. 1 / SPRING 2008 •Lloyd B and , Brodie K. (2000). Recognition of television images as a developmental milestone in young children: observational study. British Medical Journal; 320(7238): 836-8

[14] National Academy of Science,(2012). studying Media on Children and Youth, Washanton D.C

[15] 24-

[16] Royal College of Pediatrics and Child Health (2010). Safeguarding Children and Young people: roles and competences for health care staff Royal College of Pediatrics' and Child Health 2010 EC1N 8TS

[17] Robinson TN, Wilde ML, Navracruz LC, Haydel KF, Varady A (2001). Effects of reducing children's television and video game use on aggressive behavior: a randomized controlled trial. Arch Peds Adol Med. 2001;155:17-23. Discussion

[18] Sara T*, and Mats H (2011) .Mobile phone use and stress, sleep disturbances, and symptoms of depression among young adults - a prospective cohort study BMC Public Health 2011, 11:66 doi:10.1186/1471-2458-11-66

[19] Sara M, and Christina P (2008),Children and Electronic Media VOLUME 18 NUMBER 1 SPRING

[20] Strasburger V, Wilson B, Jordan A (2009). Children, Adolescents, and the Media. $2^{\text {nd }}$ ed. Thousand Oaks, CA: Sage;

[21] Smith L, Louw Q, Crous L, Grimmer-Somers K: (2009,) Prevalence of neck pain and headaches: impact of computer use and other associative factors. 29:250-257

[22] Stella D. ,Noirin H., and Brian O (2007). Center for Social and Educational Research Dublin Institute of Technology (available at ) http//arrowsdit.ie./cseerrep.

[23] (The Baby Sleep Site 2013) The Baby Sleep Site(TM), 7385 N. State Route 3 \#127, Westerville, OH 43082, USA

[24] The future of children (2000): children and computer technology. Fall/Winter The wired computer Vol. $10 \bullet$ No2

[25] Tolman D, Kim J, Schooler D, and Sorsoli C (2007).:Rethinking the associations between television viewing and adolescent sexuality development: bringing gender into focus. J Adolesc Health.; 40(1): 84.e9-84.e16

[26] Thomas C,( 1998) The Impact of Media and Technology in Schools A Research Report prepared for The Bertelsmann Foundation

[27] Woodard, E., and Gridina, N. (2000).Media in the home: The fifth annual survey of parents and children. Philadelphia: Annenberg Public Policy Center, University of Pennsylvani 